\title{
Identification of human hnRNP C1/C2 as a dengue virus NS1-interacting protein
}

\author{
Sansanee Noisakran ${ }^{\text {a,b,1 }}$, Suchada Sengsai ${ }^{\text {b,1 }}$, Visith Thongboonkerd ${ }^{\text {b,c }}$, Rattiyaporn Kanlaya ${ }^{\text {b,c }}$, \\ Supachok Sinchaikul ${ }^{d}$, Shui-Tein Chen ${ }^{\text {d,e }}$, Chunya Puttikhunt ${ }^{\mathrm{a}, \mathrm{b}}$, Watchara Kasinrerk ${ }^{\mathrm{a}, \mathrm{f}}$, \\ Thawornchai Limjindaporn $^{\mathrm{b}}$, Wiyada Wongwiwat ${ }^{\mathrm{b}}$, Prida Malasit ${ }^{\mathrm{a}, \mathrm{b}}$, Pa-thai Yenchitsomanus ${ }^{\mathrm{a}, \mathrm{b}, *}$ \\ ${ }^{a}$ Medical Biotechnology Unit, National Center for Genetic Engineering and Biotechnology, National Science and Technology Development Agency, Pathumthani 12120, Thailand \\ ${ }^{\mathrm{b}}$ Medical Molecular Biology Unit, Department of Research and Development, Faculty of Medicine Siriraj Hospital, Adulyadejvikrom Building (12th Floor), \\ Mahidol University, Bangkok 10700, Thailand \\ ${ }^{\mathrm{c}}$ Medical Proteomics Unit, Department of Research and Development, Faculty of Medicine Siriraj Hospital, Mahidol University, Bangkok 10700, Thailand \\ ${ }^{\mathrm{d}}$ Institute of Biological Chemistry and Genomic Research Center, Academia Sinica, Taipei, Taiwan \\ ${ }^{\mathrm{e}}$ Institute of Biochemical Sciences, College of Life Science, National Taiwan University, Taipei, Taiwan \\ ${ }^{\mathrm{f}}$ Department of Clinical Immunology, Faculty of Associated Medical Sciences, Chiang Mai University, Chiang Mai 50200, Thailand
}

\section{A R T I C L E I N F O}

Article history:

Received 22 April 2008

Available online 8 May 2008

\section{Keywords:}

hnRNP C1/C2

Dengue virus

NS1

Interacting protein

Host and viral protein interaction

Virus replication

\begin{abstract}
A B S T R A C T
Dengue virus nonstructural protein 1 (NS1) is a key glycoprotein involved in the production of infectious virus and the pathogenesis of dengue diseases. Very little is known how NS1 interacts with host cellular proteins and functions in dengue virus-infected cells. This study aimed at identifying NS1-interacting host cellular proteins in dengue virus-infected cells by employing co-immunoprecipitation, two-dimensional gel electrophoresis, and mass spectrometry. Using lysates of dengue virus-infected human embryonic kidney cells (HEK 293T), immunoprecipitation with an anti-NS1 monoclonal antibody revealed eight isoforms of dengue virus NS1 and a 40-kDa protein, which was subsequently identified by quadrupole time-of-flight tandem mass spectrometry (Q-TOF MS/MS) as human heterogeneous nuclear ribonucleoprotein (hnRNP) C1/C2. Further investigation by co-immunoprecipitation and co-localization confirmed the association of hnRNP C1/C2 and dengue virus NS1 proteins in dengue virus-infected cells. Their interaction may have implications in virus replication and/or cellular responses favorable to survival of the virus in host cells.
\end{abstract}

(c) 2008 Elsevier Inc. All rights reserved.

\section{Introduction}

Dengue virus is a mosquito-borne human pathogen which causes a serious public health concern around the world with approximately 100 million cases of dengue infection and 500,000 cases of hospitalizations per annum [1]. The fatality rate of the affected individuals is about $1-5 \%$ and occurs mostly in children [1]. However, the mechanisms involved in the pathogenesis of dengue hemorrhagic fever (DHF) and dengue shock syndrome (DSS) remain unraveled.

Dengue virus is a positive, single-stranded RNA virus in the genus Flavivirus of the family Flaviviridae and contains a 11-kb genome encoding three-structural proteins (capsid, C; premembrane, prM; and envelope, E) and seven-nonstructural proteins (NS1,

\footnotetext{
* Corresponding author. Address: Medical Molecular Biology Unit, Department of Research and Development, Adulyadejvikrom Buliding (12th Floor), Faculty of Medicine Siriraj Hospital, Mahidol University, Bangkok 10700, Thailand. Fax: +66 2 4184793.

E-mail address: grpye@mahidol.ac.th (P.-t. Yenchitsomanus).

1 These authors contributed equally to this work.
}

NS2A, NS2B, NS3, NS4A, NS4B, and NS5) [2]. In virus-infected cells, newly synthesized NS1 appears as a monomer in the lumen of the endoplasmic reticulum (ER) and subsequently undergoes glycosylation and dimerization as the protein is transported along the host secretory pathway to the cell surface and eventually to the extracellular milieu [3-5]. The exact roles of NS1 in each compartment are not clearly understood.

Secreted NS1 is found to activate complements in the presence or absence of specific antibodies and interact with human complement regulatory protein clusterin, potentially leading to viral and host immune complex formation and subsequent plasma leakage $[6,7]$. The correlation between levels of secreted NS1 and disease severity has also been observed $[7,8]$. Unlike the secreted form, cell surface-associated NS1 requires cross-linking of specific antibodies to induce efficient complement activation and intracellular signal transduction in response to dengue virus infection $[3,7]$.

How the NS1 molecule functions inside virus-infected cells is still elusive. A number of previous studies propose the role of intracellular NS1 in the maturation process of dengue virus [9-11]. The NS1 molecule co-localizes with double-stranded dengue viral RNA (dsRNA), and associates with intracellular membrane structures, 
which are presumed sites of virus replication, and possibly with other viral nonstructural proteins, including NS2A, NS3, NS4A, and NS5 to form viral replication complexes in virus-infected cells [9-12]. Very little is known about the interplay between dengue virus NS1, host proteins, and cellular responses during dengue virus infection. We therefore hypothesized that the intracellular NS1 may interact with host cellular proteins to facilitate its proper folding, trafficking and/or to promote favorable environment for virus production in the host cell. Biochemical and proteomic approaches were utilized in this study to identify NS1interacting proteins and subsequently confirm the protein-protein interaction.

\section{Materials and methods}

Cell line, virus, and antibodies. A human embryonic kidney epithelial cell line, 293T, dengue virus serotype 2 strain 16681, and mouse monoclonal antibodies recognizing linear epitopes (1B2, $\mathrm{NS} 1-1 \mathrm{~F}, \mathrm{NS} 1-3 \mathrm{~F}$, and NS1-4F) or conformational epitopes (NS18.2 and $1 \mathrm{~A} 4$ ) on dengue virus NS1 were obtained as described previously [13-15]. A mouse anti-human hnRNP C1/C2 monoclonal antibody (clone 4F4) was purchased from Santa Cruz Biotechnology, Inc., CA, USA.

Dengue virus infection and immunoprecipitation. 293T cells were infected with dengue virus at a multiplicity of infection (MOI) of 1 and harvested at indicated time points post-infection to verify the percentage of dengue virus infection by immunofluorescence staining for viral E and NS1 antigen expression and flow cytometry [15]. Mock-infected cells served as negative controls. Clear lysates were prepared from mock and dengue virus-infected cells and then subjected to immunoprecipitation using a mouse isotype-matched control IgG1 or IgG2a antibody (MOPC 21 or UPC 10, Sigma, St. Loius, MO, USA), a mouse anti-NS1 monoclonal antibody (1A4, IgG2a), or a mouse anti-human hnRNP C1/C2 (4F4, IgG1) according to a previously described method with minor modifications [15].

Two-dimensional (2-D) gel electrophoresis and mass spectrometry. Immunoprecipitated proteins $(200 \mu \mathrm{g})$ were subjected to the first dimensional separation by isoelectric focusing (IEF) on Immobiline DryStrip (nonlinear pH 3-10, 7-cm long; Amersham Bioscience) and the second dimensional separation in $12 \%$ SDS-polyacrylamide gel [16]. Separated protein spots were visualized by staining with Coomassie brilliant blue R-250 and the protein spot of interest was excised and in-gel digested with trypsin. Peptides were extracted from the gel pieces, purified, and analyzed by Q-TOF Ultima mass spectrometer (Micromass, Manchester, UK) according to the methods described previously [16].

Immunoblot analysis. Immunoprecipitated proteins, which had been heated or left unheated at $95{ }^{\circ} \mathrm{C}$ for $5 \mathrm{~min}$ in the presence or absence of $5 \%$ mercaptoethanol, were separated by electrophoresis in $10 \%$ SDS-polyacrylamide gel and transferred to a PVDF membrane (Millipore Corporation, Billerica, USA) using a SemiPhor semi-dry transphor unit (Amersham Bioscience). The membrane was processed as described previously [15], except that a mixture of mouse anti-NS1 monoclonal antibodies described above or a mouse anti-human hnRNP C1/C2 monoclonal antibody (4F4) was utilized in this study. The immunoreactive proteins were visualized by Western Lightning Chemiluminescence Reagent Plus (Perkin-Elmer Applied Biosystems, Foster City, CA, USA).

Double immunofluorescence staining. Mock and dengue virus-infected cells on glass coverslips were fixed with $3.7 \%$ formaldehyde in PBS for $7 \mathrm{~min}$ followed by absolute methanol for $10 \mathrm{~min}$ at room temperature (RT). The cells were incubated with a mouse isotypematched control IgG1 antibody (MOPC 21) or a mouse anti-human hnRNP C1/C2 monoclonal antibody (4F4) at the dilution of 1:500 for $1 \mathrm{~h}$ at RT. Successive incubations of the cells for $30 \mathrm{~min}$ at RT in the dark were then performed using a Cy3-conjugated rabbit anti-mouse IgG antibody (Jackson ImmunoResearch Laboratories, Inc., West Grove, PA, USA) at the dilution of 1:4000, 10\% normal mouse serum in PBS, and $20 \mu \mathrm{g} / \mathrm{ml}$ of a FITC-conjugated mouse anti-NS1 monoclonal antibody (1A4). The stained cells were visualized under a laser-scanning confocal microscope (LSM 510 Meta, Carl Zeiss, Jena, Germany).

\section{Results and discussion}

Determination of a suitable condition for preparation of dengue virusinfected cell lysates

Initially, HEK 293T cell line that had been infected with dengue virus at an MOI of 1 were collected daily for 3 days and assessed for

\section{A}

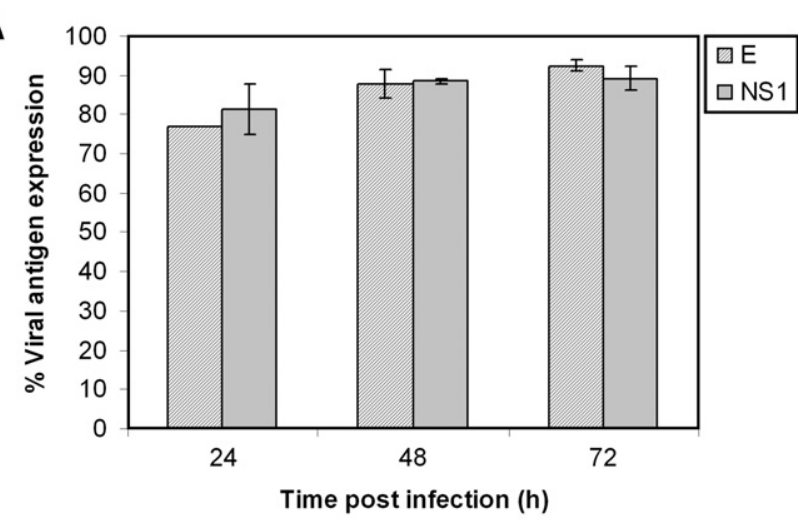

B
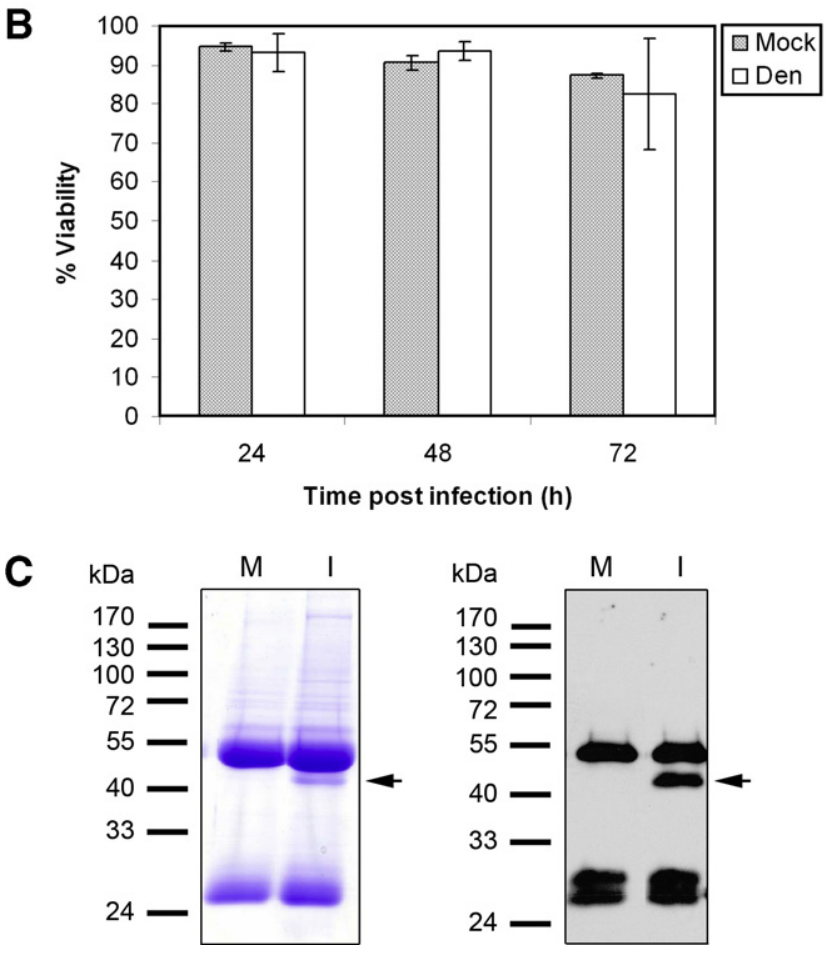

Fig. 1. Dengue virus infection of HEK 293T cells. Mock and virus-infected cells were assessed for (A) the expression of viral E and NS1 by immunofluorescence staining and flow cytometry and (B) the percentage of cell viability by trypan blue exclusion. Mock (M) and dengue virus-infected (I) cell lysates were subjected to immunoprecipitation using an anti-NS1 monoclonal antibody. Precipitated proteins were electrophoresed in a $10 \%$ SDS-polyacrylamide gel under reducing and heated conditions and visualized by Coomassie blue staining (C, left panel) or immunoblot analysis probed with an anti-NS1 specific antibody (C, right panel). Arrows indicate the dengue virus NS1 protein. 
the percentage of dengue virus infection and cell viability. Mockinfected cells served as negative controls. Infection of HEK 293T cells with dengue virus for $48 \mathrm{~h}$ resulted in high levels of viral antigen expression (Fig. 1A) but low percentage of cell death (Fig. 1B). As a result, we employed this condition for preparing cell lysates and tested whether dengue virus NS1 could be immunoprecipitated from these samples using an anti-NS1 monoclonal antibody.

Analysis by SDS-PAGE and Coomassie blue staining revealed that, under reducing and heated conditions, a unique $46-\mathrm{kDa}$ protein band was detectable in the precipitated sample from virus-infected cell lysate (Fig. 1C, left panel, lane I), but not in that from mock control (Fig. 1C, left panel, lane M). The reactivity with the anti-NS1 antibody in immunoblot analysis (Fig. 1C, right panel, lane I) strongly indicated that the specific $46-\mathrm{kDa}$ protein was monomeric NS1 of dengue virus. Two additional protein bands of about 25 and $50 \mathrm{kDa}$ were also observed in the precipitated samples from mock and dengue virus-infected cell lysates (Fig. 1C, left panel, lanes $\mathrm{M}$ and $\mathrm{I}$ ), corresponding to light and heavy chains of IgG used for the immunoprecipitation (Fig. 1C, right panel, lanes $\mathrm{M}$ and $\mathrm{I}$ ). These results therefore demonstrated that the infecting condition used in this study was adequate for establishment of dengue virus-infected samples for immunoprecipitation.
Identification of dengue virus NS1-interacting proteins by 2-D gel electrophoresis and mass spectrometry

Our results of SDS-PAGE could not show clearly any other proteins that were co-precipitated with dengue virus NS1 following immunoprecipitation with the specific antibody. This may have been due to insufficient amounts of the starting materials used for immunoprecipitation and/or the limited ability of SDS-PAGE to separate proteins with similar molecular sizes. We therefore prepared large amounts of mock and dengue virus-infected cell lysates, utilized them for immunoprecipitation using an isotypematched control antibody or an anti-NS1 monoclonal antibody, and analyzed precipitated proteins by 2-D gel electrophoresis.

Immunoprecipitation of dengue virus-infected cell lysate with the isotype-matched control antibody resulted in the appearance of two major sets of protein spots of about 25 and $50 \mathrm{kDa}$ and other minor protein spots on the 2-D SDS-polyacrylamide gel with similar patterns observed in the mock-infected cell lysate precipitated with the anti-NS1 antibody [Fig. 2A, Mock (anti-NS1) and DENV-2 (control $\lg G$ )]. Immunoblot analysis of the 25- and 50-kDa protein spots using rabbit anti-mouse immunoglobulin antibody suggested that these proteins may correspond to different isoforms

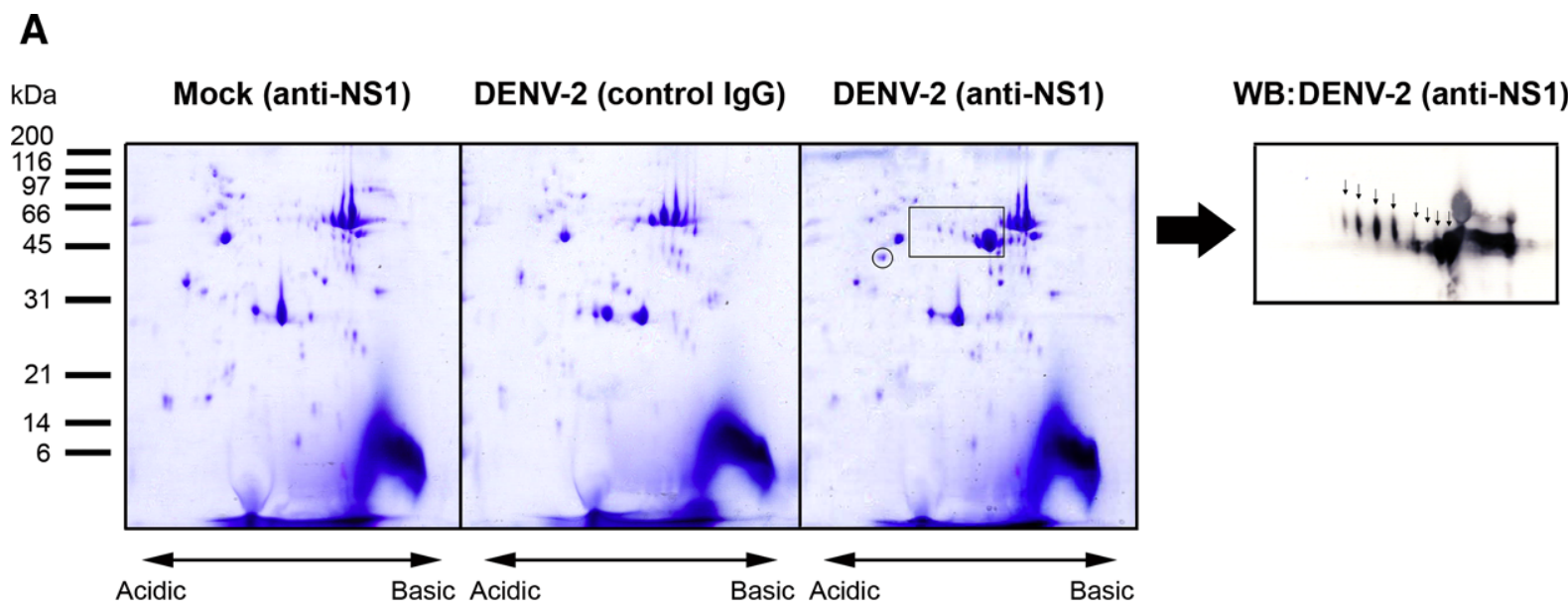

B

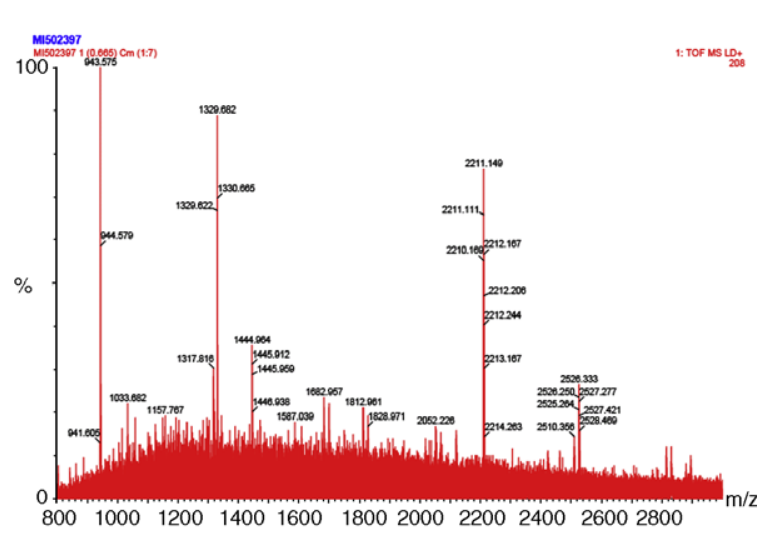

C

Heterogeneous nuclear ribonucleoproteins C1/C2 (hnRNP C1/C2) Homo sapiens (Protein ID: HNRPC_HUMAN)

lon score $=218(p<0.05)($ a significant score must be $>44)$ Method: MS/MS

Sequence coverage $13 \%$

5 peptides were identified:

VPPPPPIAR

VFIGNLNTLVVK

GFAFVQYVNER

VFIGNLNTLVVKK

IVGCSVHKGFAFVQYVNER

Whole sequence of this protein:

1 MASNVTNKTD PRSMNSRVFI GNLNTLVVKK SDVEAIFSKY GKIVGCSVHK

51 GFAFVOYVNE RNARAAVAGE DGRMIAGQVL DINLAAEPKV NRGKAGVKRS

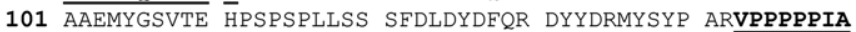

151 RAVVPSKRQR VSGNTSRRGK SGFNSKSGQR GSSKSGKLKG DDLQAIKKEL

201 TOIKOKVDSL LENLEKMEKE OSKOAVEMKN DKSEEEOSSS SVKKDETNVK

251 MESEGGADDS AEEGDLLDDD DNEDRGDDQL ELIKDDEKEA EEGEDDRDSA 301 NGEDDS

$\mathrm{pH} 5.0$

Molecular mass $33.73 \mathrm{kDa}$

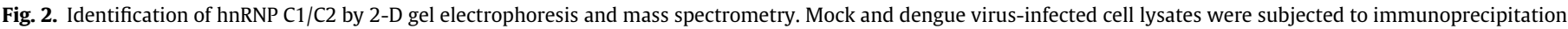

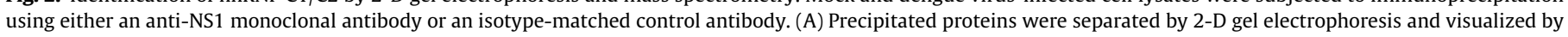

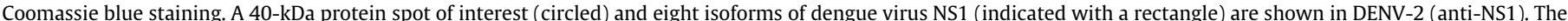

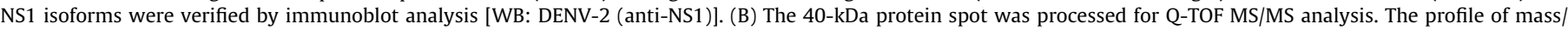

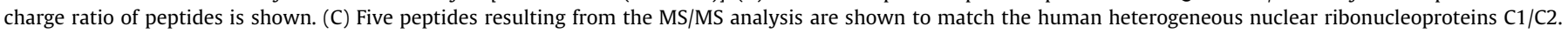


of IgG light and heavy chains, respectively (data not shown). Apart from the detection of these protein spots, immunoprecipitation of dengue virus-infected cell lysate with the anti-NS1 specific antibody yielded a unique profile of eight protein spots of approximately $46 \mathrm{kDa}$ as well as a single $40-\mathrm{kDa}$ protein spot [Fig. 2, DENV-2 (anti-NS1)] which were not detectable in other precipitated control samples [Fig. 2, Mock (anti-NS1) and DENV-2 (control $\operatorname{IgG})]$. The former set of protein spots likely represented various isoforms of dengue virus NS1 owing to their reactivity with an anti-NS1 antibody in the immunoblot analysis [Fig. 2A-the rightmost inset, WB: DENV-2 (anti-NS1)]. The unknown 40-kDa protein spot, which was co-precipitated with dengue virus NS1, was subsequently excised, in-gel digested with trypsin and processed for mass spectrometry. Spectra of the eluting peptides obtained from mass spectrometric analysis were shown in Fig. 2B. Five peptides were identified and, based on the SwissProt database, were deduced to be from human heterogeneous nuclear ribonucleoproteins (hnRNP) C1/C2 (SwissProt accession number P07910) (Fig. 2C). The identified peptides were common to both isoforms of hnRNP $C$ and corresponded to sequence coverage of about $13 \%$ of the protein (Fig. 2C).

\section{Co-immunoprecipitation of hnRNP C1/C2 and dengue virus NS1}

Human hnRNP C1/C2 are members of the heterogeneous nuclear ribonucleoprotein family which consists of 20 major hnRNP proteins (designated hnRNP A1 through $U$ ) with molecular sizes of approximately $36-120 \mathrm{kDa}[17,18]$. Alternative mRNA splicing of hnRNP C proteins with a 13-amino acid deletion occurring after glycine 106 or serine 107 generates two isoforms of proteins, hnRNP C1 (290 amino acids) and hnRNP C2 (303 amino acids) $[19,20]$. The hnRNP C1 and C2 proteins (41 and $43 \mathrm{kDa}$ ) are involved in mRNA biogenesis and contain important conserved motifs essential for RNA binding, protein-protein interaction and nuclear localization $[17,18,21-23]$. In order to determine whether the hnRNP C1/C2 interacts with dengue virus NS1 in virus-infected cells, immunoprecipitation of mock and dengue virus-infected cell lysates was performed using isotype-matched control antibodies or monoclonal antibodies specific against human hnRNP C1/C2 or dengue virus NS1. The presence of the two proteins in each precipitated sample was then analyzed by the immunoblot assay under nonreducing and unheated conditions. The lysates prior to immunoprecipitation were also included in this assay as controls for detection of hnRNP C1/C2 and dengue virus NS1.

As expected, hnRNP C1/C2 was observed in mock and dengue virus-infected cell lysate while dengue virus NS1 was detected only in the latter sample (Fig. 3, input). Using the anti-NS1 monoclonal antibody for immunoprecipitation, dengue virus NS1 and hnRNP C1/C2 were pulled down from virus-infected lysate, but not from mock-infected control (Fig. 3, IP: $\alpha \mathrm{NS} 1$ ). On the contrary, immunoprecipitation with the anti-hnRNP C1/C2 monoclonal antibody yielded only the hnRNP C1/C2 in mock and dengue virus-infected samples, but no NS1 dimer was observed (Fig. 3, IP: ahnRNP). As negative controls, immunoprecipitation with either isotype-matched control antibody did not give any specific band of NS1 dimer; however, relatively low levels of hnRNP C1/C2 could be detected probably as a result of certain extents of nonspecific binding to protein G-Sepharose beads (Fig. 3, IP: IgG1 and IgG2a). Taken together, these findings suggested that the interaction between the dengue NS1 and hnRNP C1/C2 occurred in virus-infected cells. Inability to co-immunoprecipitate dengue virus NS1 using the anti-hnRNP C1/C2 antibody could be due to either masking of the hnRNP $\mathrm{C} 1 / \mathrm{C} 2$ epitope by the interacting protein as suggested by a previous work on the Epstein-Barr virus SM protein [24], or only a small portion of the total hnRNP C1/C2 population involved in the dengue virus NS1 interaction.

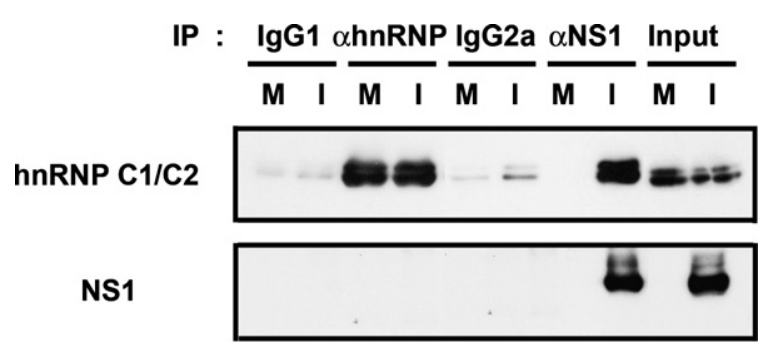

Fig. 3. Co-immunoprecipitation of hnRNP C1/C2 and dengue virus NS1. Mock (M) and dengue virus-infected (I) cell lysates were processed for immunoprecipitation (IP) using isotype-matched control antibodies (IgG1 or IgG2a), an anti-hnRNP C1/C2 monoclonal antibody (clone 4F4, IgG1), or an anti-NS1 monoclonal antibody (clone $1 \mathrm{~A} 4, \mathrm{IgG} 2 \mathrm{a}$ ). Each precipitated sample was detected for the presence of hnRNP C1/ C2 and dengue virus NS1 by immunoblot analysis using specific antibodies. Mock and virus-infected cell lysates prior to the immunoprecipitation (input) served as controls for the protein detection.

\section{Co-localization of hnRNP C1/C2 and dengue virus NS1}

Further investigation was carried out to determine sub-cellular distribution of hnRNP C1/C2 and dengue virus NS1 by double immunofluorescence staining and confocal microscopy. hnRNP $\mathrm{C} 1 / \mathrm{C} 2$ was found predominantly in the nucleus and, to a lesser extent, in the cytoplasm of both mock (data not shown) and dengue virus-infected cells with an evenly distributed pattern (Fig. 4A and $\mathrm{B}$, hnRNP $\mathrm{C} 1 / \mathrm{C} 2$ ). Although hnRNP $\mathrm{C} 1 / \mathrm{C} 2$ is primarily a nuclear protein in the cells, it can be translocated to the cytoplasm following the induction of apoptosis via Rho-activated kinase activation [25] or during poliovirus and human rhinovirus infection through an apoptosis-independent mechanism [26,27]. Nevertheless, an increase in the nuclear efflux of the hnRNP C1/C2 was not observed upon dengue virus infection in our study (data not shown).

Unlike the hnRNP $\mathrm{C} 1 / \mathrm{C} 2$, dengue virus NS1 was detected as large foci mainly in the cytoplasm of dengue virus-infected cells (Fig. 4A and B, NS1), consistent with the staining pattern observed in previous studies $[7,10,15]$. A partial co-localization of the hnRNP C1/C2 and NS1 was detectable in some dengue virus-infected cells particularly at the perinuclear regions (Fig. $4 \mathrm{~A}$ and $\mathrm{B}$, Merge). This observation may help to explain the incapability to detect reciprocal co-immunoprecipitation with the anti-hnRNP C1/C2 antibody (Fig. 3, IP: ahnRNP). Consistent findings on the co-localization and the co-immunoprecipitation of hnRNP $\mathrm{C} 1 / \mathrm{C} 2$ with dengue virus NS1 was observed not only in HEK 293T cell line (Fig. 3 and 4) but also in other three human cell lines (including liver HepG2, fibroblast HF and endothelial EAhy926) and primary human umbilical vein endothelial cells, HUVEC, (data not shown), confirming that this interaction is genuine, but probably transient and involved in only a part of total host cellular hnRNP C1/C2.

A previous study using a yeast two hybrid system identified human signal transducer and activator of transcription $3 \beta$ (STAT3 $\beta$ ) as a dengue virus NS1-interacting protein which may be implicated in the induction of IL-6 levels and subsequent plasma leakage [28]. By utilizing different approach, we demonstrated herein that hnRNP $\mathrm{C} 1 / \mathrm{C} 2$ is additional host cellular protein that associates with dengue virus NS1 in virus-infected cells, but mechanisms of this protein-protein interaction are not known. The common structural motifs on hnRNP C1/C2 $[17,22,23]$ may interact with dengue virus NS1 directly or indirectly through a link of other host proteins, viral proteins, and/or viral RNA resulting in the formation of viral replication complexes. This notion was supported by a previous study on the role of hnRNP C1 in the initiation of positive-strand viral RNA synthesis in poliovirus-infected cells through its interaction with the 3 '-end of negative strand RNA of poliovirus and other three viral protein precursors, polypeptide $3 \mathrm{CD}, \mathrm{P} 2$, and $\mathrm{P} 3$, crucial for poliovirus replication [29]. In addition, detection of various 
A

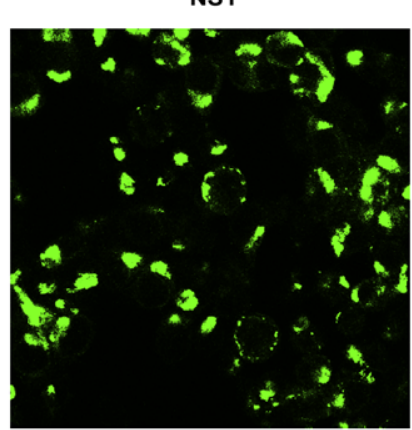

hnRNP C1/C2

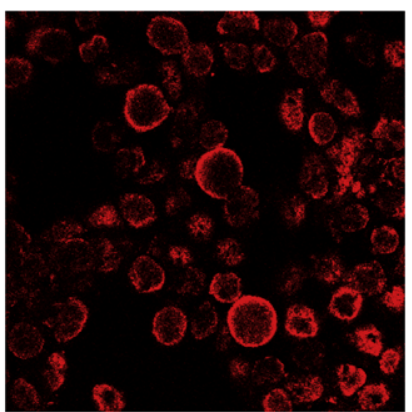

B
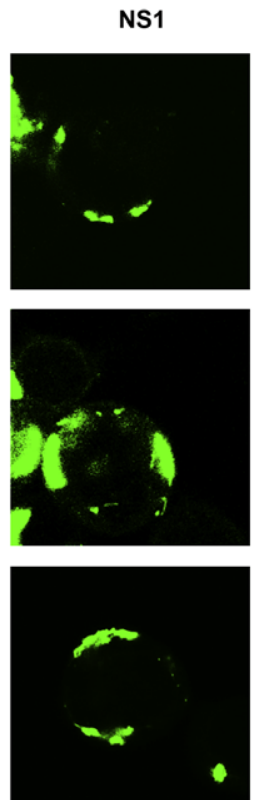

hnRNP C1/C2
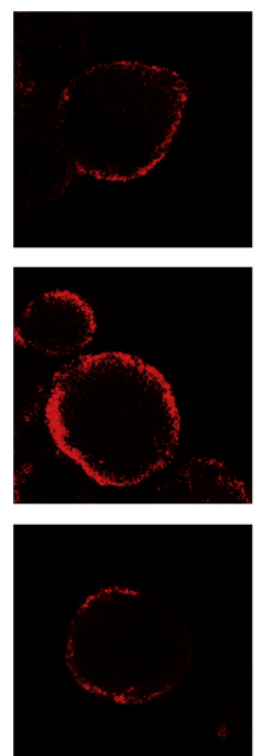

Bright field

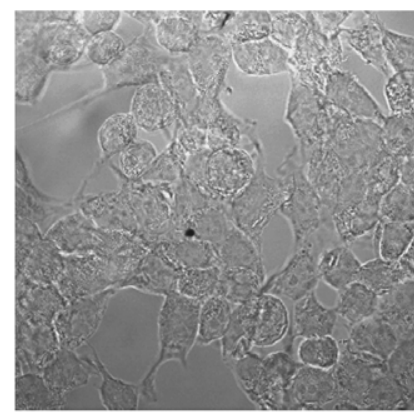

Merge
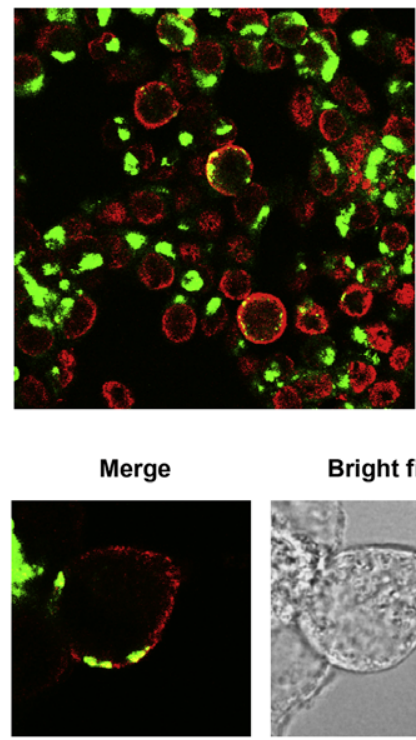

Bright field
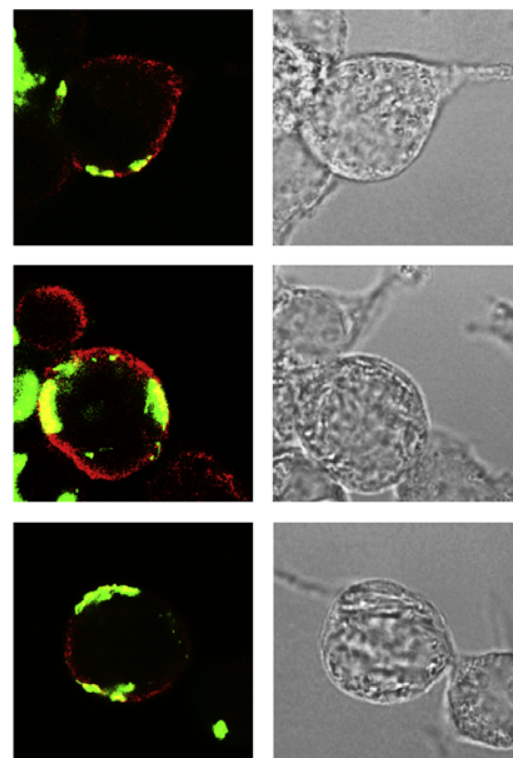

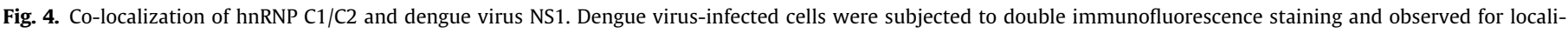

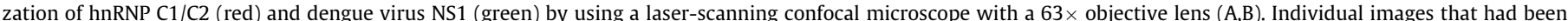

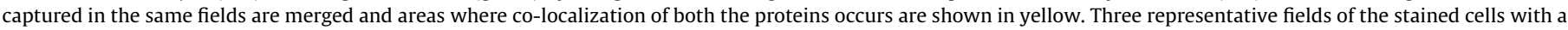
$5 \times$ magnification are shown in (B). (For interpretation of the references to color in this figure legend, the reader is referred to the web version of this paper.)

isoforms of dengue virus NS1 with distinct isoelectric points $(\mathrm{p} I)$ by 2-D gel electrophoresis in our study implied that differential posttranslational modifications of NS1 might occur in virus-infected cells and give rise to generation of different isoforms of the protein, each of which potentially exerts different functions. Which particular isoform of dengue virus NS1 interacts with hnRNP C1/C2 and whether this association is involved in virus replication and host cellular responses during dengue virus infection require further investigations.

\section{Acknowledgments}

We thank Dr. Panisadee Avirutnan for technical assistance in HUVEC cultures. This work was supported by a research grant (BT-B-02-MG-B4-4801) from the National Center for Genetic Engineering and Biotechnology (to S.N.), Senior Research Scholar Grants from Thailand Research Fund (to P.M. and P.Y.), and Siriraj Graduate Thesis Scholarship from the Faculty of Medicine Siriraj Hospital, Mahidol University (to S.Se.). 


\section{References}

[1] World Health Organization, Dengue and dengue haemorrhagic fever. <http:// www.who.int/mediacentre/factsheets/fs117/en/>, 2008 (accessed April 7).

[2] B.D. Lindenbach, C.M. Rice, Flaviviridae: the viruses and their replication, in: D.M. Knipe, P.M. Howley, D.E. Griffin, R.A. Lamb, M.A. Martin, B. Roizman, S.E. Strauss (Eds.), Fields Virology, fourth ed., Lippincott Williams \& Wilkins, Philadelphia, 2001, pp. 991-1041.

[3] M.G. Jacobs, P.J. Robinson, C. Bletchly, J.M. Mackenzie, P.R. Young, Dengue virus nonstructural protein 1 is expressed in a glycosylphosphatidylinositol-linked form that is capable of signal transduction, FASEB J. 14 (2000) 1603-1610.

[4] G. Winkler, V.B. Randolph, G.R. Cleaves, T.E. Ryan, V. Stollar, Evidence that the mature form of the flavivirus nonstructural protein NS1 is a dimer, Virology 162 (1988) 187-196.

[5] M. Flamand, F. Megret, M. Mathieu, J. Lepault, F.A. Rey, V. Deubel, Dengue virus type 1 nonstructural glycoprotein NS1 is secreted from mammalian cells as a soluble hexamer in a glycosylation-dependent fashion, J. Virol. 73 (1999) 6104-6110.

[6] T. Kurosu, P. Chaichana, M. Yamate, S. Anantapreecha, K. Ikuta, Secreted complement regulatory protein clusterin interacts with dengue virus nonstructural protein 1, Biochem. Biophys. Res. Commun. 362 (2007) 10511056.

[7] P. Avirutnan, N. Punyadee, S. Noisakran, C. Komoltri, S. Thiemmeca, K. Auethavornanan, A. Jairungsri, R. Kanlaya, N. Tangthawornchaikul, C. Puttikhunt, S. Pattanakitsakul, P. Yenchitsomanus, J. Mongkolsapaya, W. Kasinrerk, N. Sittisombut, M. Husmann, M. Blettner, S. Vasanawathana, S. Bhakdi, P. Malasit, Vascular leakage in severe dengue virus infections: a potential role for the non-structural viral protein NS1 and complement, J. Infect. Dis. 193 (2006) 1078-1088.

[8] D.H. Libraty, P.R. Young, D. Pickering, T.P. Endy, S. Kalayanarooj, S. Green, D.W. Vaughn, A. Nisalak, F.A. Ennis, A.L. Rothman, High circulating levels of the dengue virus nonstructural protein NS1 early in dengue illness correlate with the development of dengue hemorrhagic fever, J. Infect. Dis. 186 (2002) 11651168.

[9] B.D. Lindenbach, C.M. Rice, Genetic interaction of flavivirus nonstructural proteins NS1 and NS4A as a determinant of replicase function, J. Virol. 73 (1999) 4611-4621

[10] J.M. Mackenzie, M.K. Jones, P.R. Young, Immunolocalization of the dengue virus nonstructural glycoprotein NS1 suggests a role in viral RNA replication, Virology 220 (1996) 232-240.

[11] A.A. Khromykh, P.L. Sedlak, E.G. Westaway, cis- and trans-acting elements in flavivirus RNA replication, J. Virol. 74 (2000) 3253-3263.

[12] E.G. Westaway, J.M. Mackenzie, M.T. Kenney, M.K. Jones, A.A. Khromykh, Ultrastructure of Kunjin virus-infected cells: colocalization of NS1 and NS3 with double-stranded RNA, and of NS2B with NS3, in virus-induced membrane structures, J. Virol. 71 (1997) 6650-6661.

[13] C. Puttikhunt, W. Kasinrerk, S. Srisa-ad, T. Duangchinda, W. Silakate, S. Moonsom, N. Sittisombut, P. Malasit, Production of anti-dengue NS1 monoclonal antibodies by DNA immunization, J. Virol. Methods 109 (2003) 55-61.
[14] K. Sittisiri, Production and characterization of monoclonal antibodies to dengue 2 virus, M.Sc. Thesis, Mahidol University, 1994

[15] S. Noisakran, T. Dechtawewat, P. Rinkaewkan, C. Puttikhunt, A Kanjanahaluethai, W. Kasinrerk, N. Sittisombut, P. Malasit, Characterization of dengue virus NS1 stably expressed in 293T cell lines, J. Virol. Methods 142 (2007) 67-80

[16] S.N. Pattanakitsakul, K. Rungrojcharoenkit, R. Kanlaya, S. Sinchaikul, S. Noisakran, S.T. Chen, P. Malasit, V. Thongboonkerd, Proteomic analysis of host responses in HepG2 cells during dengue virus infection, J. Proteome Res. 6 (2007) 4592-4600.

[17] G. Dreyfuss, M.J. Matunis, S. Pinol-Roma, C.G. Burd, hnRNP proteins and the biogenesis of mRNA, Annu. Rev. Biochem. 62 (1993) 289-321.

[18] S. Pinol-Roma, HnRNP proteins and the nuclear export of mRNA, Semin. Cell Dev. Biol. 8 (1997) 57-63.

[19] C.G. Burd, M.S. Swanson, M. Gorlach, G. Dreyfuss, Primary structures of the heterogeneous nuclear ribonucleoprotein A2, B1, and C2 proteins: a diversity of RNA binding proteins is generated by small peptide inserts, Proc. Natl. Acad. Sci. USA 86 (1989) 9788-9792.

[20] M.S. Swanson, T.Y. Nakagawa, K. LeVan, G. Dreyfuss, Primary structure of human nuclear ribonucleoprotein particle $C$ proteins: conservation of sequence and domain structures in heterogeneous nuclear RNA, mRNA, and pre-rRNA-binding proteins, Mol. Cell. Biol. 7 (1987) 1731-1739.

[21] J.H. Kim, B. Hahm, Y.K. Kim, M. Choi, S.K. Jang, Protein-protein interaction among hnRNPs shuttling between nucleus and cytoplasm, J. Mol. Biol. 298 (2000) 395-405.

[22] B. Carpenter C. MacKay, A Alnabulsi, M. MacKay, C. Telfer, WT Melvin, G. Murray, The roles of heterogeneous nuclear ribonucleoproteins in tumour development and progression, Biochim. Biophys. Acta 1765 (2006) 85-100.

[23] M. Gorlach, C.G. Burd, G. Dreyfuss, The determinants of RNA-binding specificity of the heterogeneous nuclear ribonucleoprotein C proteins, J. Biol. Chem. 269 (1994) 23074-23078

[24] S.C. Key, T. Yoshizaki, J.S. Pagano, The Epstein-Barr virus (EBV) SM protein enhances pre-mRNA processing of the EBV DNA polymerase transcript, J. Virol. 72 (1998) 8485-8492.

[25] H.H. Lee, C.L. Chien, H.K. Liao, Y.J. Chen, Z.F. Chang, Nuclear efflux of heterogeneous nuclear ribonucleoprotein $\mathrm{C} 1 / \mathrm{C} 2$ in apoptotic cells: a novel nuclear export dependent on Rho-associated kinase activation, J. Cell Sci. 117 (2004) 5579-5589.

[26] K.E. Gustin, P. Sarnow, Effects of poliovirus infection on nucleo-cytoplasmic trafficking and nuclear pore complex composition, EMBO J. 20 (2001) 240249.

[27] K.E. Gustin, P. Sarnow, Inhibition of nuclear import and alteration of nuclear pore complex composition by rhinovirus, J. Virol. 76 (2002) 8787-8796.

[28] J.J. Chua, R. Bhuvanakantham, V.T. Chow, M.L. Ng, Recombinant non-structural 1 (NS1) protein of dengue-2 virus interacts with human STAT3beta protein, Virus Res. 112 (2005) 85-94.

[29] J.E. Brunner, J.H. Nguyen, H.H. Roehl, T.V. Ho, K.M. Swiderek, B.L Semler, Functional interaction of heterogeneous nuclear ribonucleoprotein $\mathrm{C}$ with poliovirus RNA synthesis initiation complexes, J. Virol. 79 (2005) $3254-$ 3266. 